\title{
Internal Fixation with a Locking T-Plate for Proximal Humeral Fractures in Patients Aged 65 Years and Older
}

\author{
Jae-Kwang Yum ${ }^{\circledR}$, Min-Kyu Seong, Chi-Woon Hong \\ Department of Orthopedic Surgery, Inje University Sanggye Paik Hospital, Seoul, Korea
}

\begin{abstract}
Background: The purpose of this study was to evaluate the clinical and radiographic outcomes of internal fixation with locking T-plates for osteoporotic fractures of the proximal humerus in patients aged 65 years and older.

Methods: From January 2007 through to December 2015, we recruited 47 patients aged 65 years and older with osteoporotic fractures of the proximal humerus. All fractures had been treated using open reduction and internal fixation with a locking T-plate. We classified the fractures in accordance to the Neer classification system; At the final follow-up, the indicators of clinical outcome-the range of motion of the shoulder (flexion, internal rotation, and external rotation) and the presence of postoperative complications-and the indicators of radiographic outcome - the time-to-union and the neck-shaft angle of the proximal humerus - were evaluated. The Paavolainen method was used to grade the level of radiological outcome in the patients.

Results: The mean flexion was $155.0^{\circ}$ (range, $90^{\circ}-180^{\circ}$ ), the mean internal rotation was T8 (range, T6-L2), and the mean external rotation was $66.8^{\circ}$ (range, $30^{\circ}-80^{\circ}$ ). Postoperative complications, such as plate impingement, screw loosening, and varus malunion were observed in five patient. We found that all patients achieved bone union, and the mean time-to-union was 13.5 weeks of the treatment. The mean neck-shaft angle was $131.4^{\circ}$ at the 6-month follow-up. According to the Paavolainen method, "good" and "fair" radiographic results each accounted for 38 and 9 of the total patients, respectively.

Conclusions: We concluded that locking T-plate fixation leads to satisfactory clinical and radiological outcomes in elderly patients with proximal humeral fractures by providing a larger surface area of contact with the fracture and a more rigid fixation.
\end{abstract}

(Clin Shoulder Elbow 2017;20(4):217-221)

Key Words: Proximal humerus; Osteoporotic fractures; Locking T-plate; Internal fixation

\section{Introduction}

Fractures of the proximal humerus compose approximately $5 \%$ of all fractures. The prevalence of proximal humeral fractures is steadily increasing with a rapidly aging population. Compared to their younger counterparts, the older generation and in particular those that suffer from osteoporosis are more vulnerable to these fractures, because even low-energy trauma can cause fractures in this subset of population. ${ }^{1)}$ Palvanen et al. ${ }^{2)}$ reported that the number of patients with proximal humeral fractures had more than tripled between 1970 and 2002.

The majority of these fractures, which show only mild dis- placement, are conservatively treated with satisfactory clinical outcomes; however, it has been reported that approximately $20 \%$ still require surgical treatment. ${ }^{3)}$ This generally applies to older patients, who compared to younger patients have more severe comminution and poorer bone quality. These hurdles make long-term reduction, rigid fixation, and thereby successful bone union more difficult to achieve. Furthermore, elderly patients present with postoperative complications, such as bone necrosis, non- or malunion, post-trauma shoulder stiffness, and infections more often than younger patients. Researchers have developed a wide range of treatments to minimize these complications. ${ }^{4}$

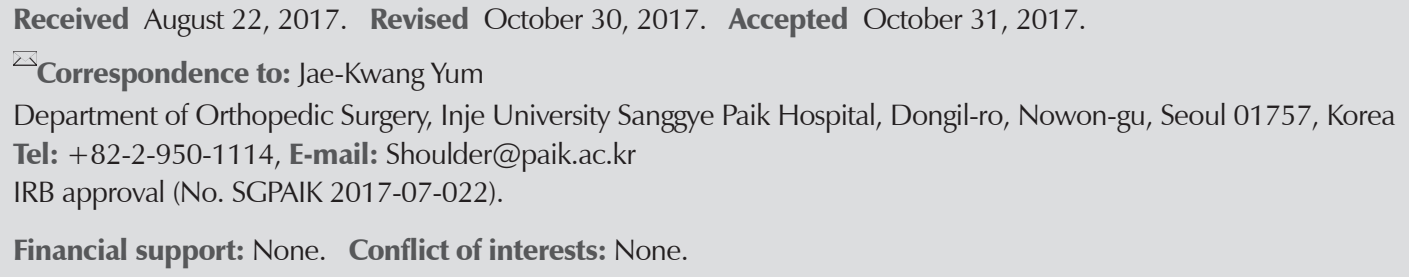


Types of surgical treatments for proximal humeral fractures include percutaneous pin fixation, fracture repair, tension band wiring, intramedullary nailing, plate fixation, and arthroplasty. The goal of surgical treatment for proximal humeral fractures is to stabilize the fracture through anatomical reduction and fixation. Factors that are considered to be important for the anatomical reduction include maintenance of the neck-shaft angle of the proximal humerus, restoration of the medial support, and stability of the reduction. ${ }^{5,6)}$ The choice of surgical treatment and the fixative device will depend on these factors along with the surgeon's level of experience and preference.

In spite of their drawbacks such as excessive incision/dissection and large amounts of bleeding rising from the treatment, open reduction and internal fixation involving plates is one of the most widely used surgical treatments for proximal humeral fractures. This is because this method enables accurate reduction and leads to minimal neural and vascular injuries. The most commonly used type of plating is the locking compression plate (LCP). Other plates include the T-shaped LCP (T-LCP), investigated in this study, as well as the blade plate and the dynamic compressing plate.

Recent studies seem to have preferentially used LCPs over other fixation devices to perform internal fixation. ${ }^{7-10)}$ This may be because LCPs compared to other devices provide more rigid fixation, by allowing multidirectional fixation, and better platescrew fixation in patients with osteoporosis. ${ }^{11)}$ However, LCPassociated complications, such as non- or malunion, plate breakage, and screw loosening have been reported. ${ }^{5}{ }^{5}$ In this study, we investigated the clinical and radiological outcomes after internal fixation using the less commonly used plate-the locking Tplate - in patients with proximal humeral fractures to evaluate their effectiveness.

\section{Methods}

We recruited 47 patients aged 65 years or older who had received treatment for a proximal humeral fracture from January 2007 through to December 2015. We categorized the patients' fractures by the Neer classification system. The presence or absence of osteoporotic fractures was determined in terms of bone density or as the presence/absence of compression fractures on spinal radiography. We excluded patients who could not be followed-up for more than 6 months.

Among the 47 patients, 14 were men and 33 were women. The mean age of the patients was 68.8 years (range, 65-85 years). According to the Neer classification, the types of fractures were two-part in 38 patients and three-part in 9. The mean follow-up period was 17.3 months (range, 9.3-93.1 months). We observed a successful bone union in all the patients (Table 1 ).

We used the deltopectoral approach in all the patients. After the anatomical reduction, we placed the T-LCP (LCP T-plate
4.5/5.0; Synthes, Solothurn, Switzerland) at the posterior bicipital groove, or at least so that the proximal and the distal fracture fragments were in contact as much as possible, and carried out screw fixation. A portable C-arm device was used, intraoperatively, to confirm the anatomical reduction of the fracture and, postoperatively, to confirm whether the fixation is maintained during joint movement and whether there is any articular invasion of the screw.

The patient was applied with a shoulder brace and allowed a range of movement below the elbow during the first 4 postoperative weeks. After which, the brace was removed and the patient began rehabilitation to enhance range of motion (ROM) of the shoulder. When the patient's shoulder ROM was restored, muscle-strengthening exercises were begun.

We evaluated the clinical outcome in terms of the shoulder ROM (flexion, internal rotation, and external rotation) and the presence/absence of postoperative complications, such as reduction loss, failure of plate fixation, screw loosening, and avascular necrosis of the humeral head at the final follow-up. We measured the radiological outcome in terms of the difference in neck-shaft angle of the proximal humerus between two timepoints: immediately postoperative and 6-month postoperative. Using the method described by Paavolainen et al., ${ }^{12)}$ we defined and calculated the proximal humeral neck-shaft angle as the angle that results from the intersection between the axial axis of the humeral shaft and the line perpendicular to the humeral tibia (Fig. 1, 2).

\section{Results}

We found that mean shoulder ROMs were $155.0^{\circ}$ for flexion (range, $90^{\circ}-180^{\circ}$ ), T8 for internal rotation (range, T6-L2), and $66.8^{\circ}$ for external rotation (range, $30^{\circ}-80^{\circ}$ ) at the final follow-up. We observed the following postoperative complications: mild pain from subacromial impingement of the plate (one patient); screw loosening (three patients); and malunion (one patient). Complications such as reduction loss, nonunion, and avascular necrosis of the humeral head were not found (Table 2).

Using radiography, we found that the mean time-to-union

Table 1. Summary of Patient Characteristics

\begin{tabular}{lc}
\hline \multicolumn{1}{c}{ Objective } & Value \\
\hline Sex (male:female) & $14: 33$ \\
\hline Age (yr) & $68.8(65-85)$ \\
\hline Neer classification & \\
\hline Two part & 38 \\
\hline Three part & 9 \\
\hline Clinical follow-up period (mo) & $17.3(9.3-93.1)$ \\
\hline
\end{tabular}

Values are presented as number only or median (range). 
was 13.5 weeks (range, 7.1-69.1 weeks) and the mean proximal humeral neck-shaft angle was $136.0^{\circ}$ (range, $111.0^{\circ}-157.1^{\circ}$ ) at the immediate-postoperative follow-up and $131.4^{\circ}$ (range, $109.9^{\circ}-150.1^{\circ}$ ) at the 6 -month follow-up, giving a mean difference in neck-shaft angle of $4.6^{\circ}$ (range, $-4.82^{\circ}-4.3^{\circ}$ ). The individual neck-shaft angles according to the method developed by Paavolainen et al. ${ }^{12)}$ ware "excellent" in thirty-eight patients and "fair" in nine.

\section{Discussion}

In this study, we found clinically and radiologically satisfactory outcomes after internal fixation using T-LCPs. Compared to T-

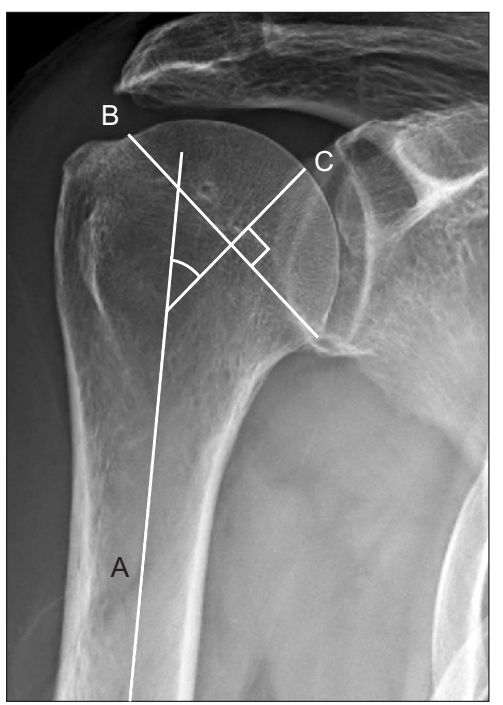

Fig. 1. Neck-shaft angle. Using an anteroposterior radiograph, the humeral neck/shaft angle was determined by the intersection of a line drawn on the central axis of the humeral shaft (A) with a line $\mathrm{C}$ drawn perpendicular to the anatomical neck (B) of the humerus.
LCPs, LCPs are smaller, have a lower rigidity and higher elasticity, and biomechanically provide earlier stability and stronger rotational stability. Treatments using LCPs have been associated with minimal injury to circulatory networks in the humeral head and a stable fixation angle. Studies have also reported that LCPs provide sustained reduction via restoration of the medial support. For these advantages, the surgical indication of LCP fixation has been preferred for patients with osteoporosis. ${ }^{10)}$ However, it has been reported that a defective plate or screw loosening leads to a greater chance of reduction loss after LCP fixation than after TLCP fixation because LCPs are thinner than T-LCPs. Moreover, varus displacements of the fracture fragment has been shown to be more prevalent with LCP than T-plate fixation. ${ }^{13)}$

Although multiple studies have shown that LCP fixation leads to satisfactory outcomes, complications associated with this treatment have also been reported. For instance, Min et al. ${ }^{6}$ reported a number of instances of failed fixations because of plate or screw loosening or plate-related defects when patients with proximal humeral fractures were treated using internal fixation

Table 2. Complications

\begin{tabular}{lc}
\hline \multicolumn{1}{c}{ Complication } & Cases \\
\hline Plate impingement & 1 \\
\hline Screws loosening & 3 \\
\hline Malunion & 1 \\
\hline Reduction loss & 0 \\
\hline Nonunion & 0 \\
\hline AVN of proximal humeral head & 0 \\
\hline Axillary nerve injury & 0 \\
\hline Total & 5 \\
\hline
\end{tabular}

AVN: avascular necrosis.
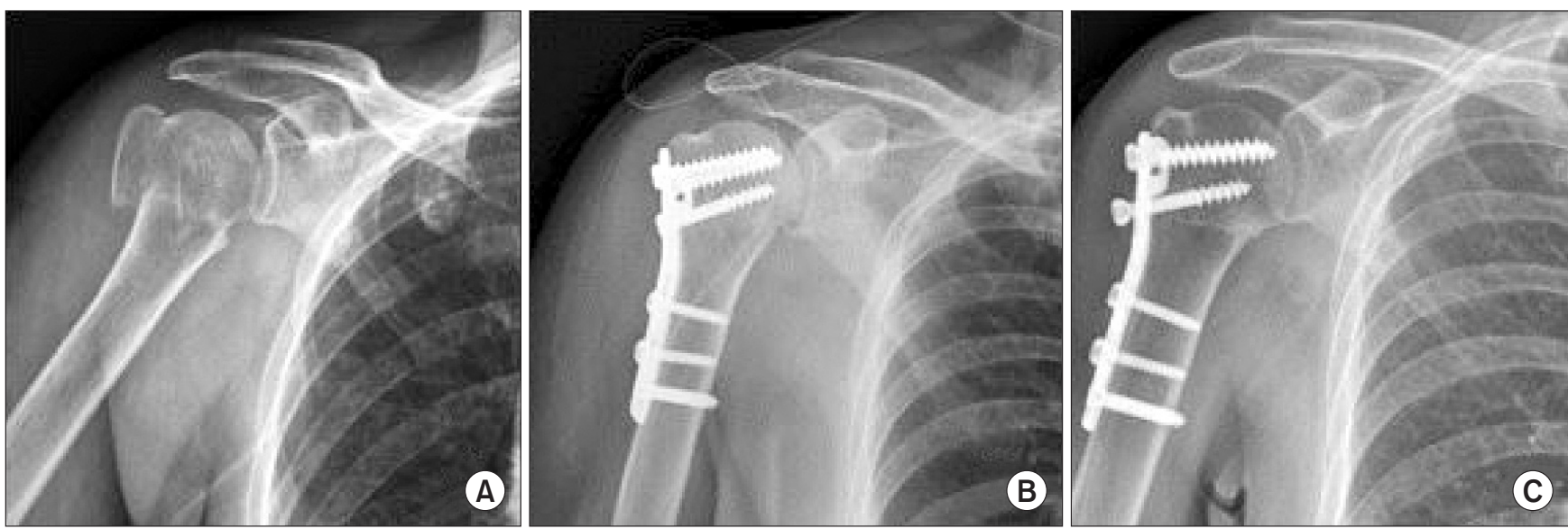

Fig. 2. (A) Preooperative radiograph of a 69-year-old female shows 2 part right proximal humerus fracture. (B) Immediate postoperative radiograph shows that neck shaft angle was $140^{\circ}$. (C) Last follow up radiograph shows that neck shaft angle was $125^{\circ}$. 
with LCPs. Some risk factors of complications suggested by the authors include a non-anatomical reduction, medial cortical bone deficit, fixation using a short locking screw at the humeral head, and inappropriate fixation of the greater tuberosity.

Bansal et al. ${ }^{14)}$ reported the functional outcomes, in terms of the Constant score, and the postoperative complications after internal fixation using LCPs for proximal humeral fractures in 25 patients. At the 6-month follow-up, the average Constant score was 57.4 , showing a generally favorable outcome in patients ("excellent" in 4 patients [16\%]; "good" in 11 patients [44\%]; "fair" in 4 patients [16\%]; and "poor" in 6 patients [24\%]). However, they also reported the following percentages of postoperative complications: infection of the area of injury in two patients $(8 \%)$, impingement in one patient $(4 \%)$, malunion in 5 patients $(20 \%)$, screw perforation in four patients $(16 \%)$, distal loosening of the screw and plate in one patient $(4 \%)$, and avascular necrosis in two patients (8\%). Thus, although internal fixation with LCPs was associated with satisfactory functional outcomes, the occurrence of complications and re-treatment rates associated with it were relatively high. The authors emphasized that the experience and expertise of the surgeon in performing the plating are vital to minimize these complications. Similarly, in another study, Sommer et al. ${ }^{15)}$ noted that key factors for a successful surgical outcome after internal fixation with LCPs are appropriate choice of plates and screws and expertise in using surgical tools.

Currently, there is no consensus as to the gold standard of treatment for proximal humeral fractures in elderly patients with severe osteoporosis. Hussain et al. ${ }^{16)}$ reported the functional outcomes and postoperative complications after internal fixation using T-LCPs in 25 patients with proximal humeral fractures after two or more years of follow-up. Based on the Neer scoring system, the functional outcome was generally favorable: "excellent" in twenty-two patients (88\%); "fair" in two patients (8\%); and "poor" in one patient (4\%). They observed postoperative complications, such as superficial skin infection in three patients (12\%); screw loosening, discovered during the rehabilitation period, in two patients (8\%); and avascular necrosis in one patient $(4 \%)$. They suggested that for a successful outcome the following criteria should be met: 1) the appropriate treatment indication for patients, 2) expertise in using surgical devices, and 3) patient compliance to rehabilitation protocols. They also recommended the fulfillment of the following procedural precautions during surgery: 1) avoid impingement during shoulder abduction by placing the plate $7-8 \mathrm{~mm}$ below the greater tuberosity; 2 ) avoid intraarticular invasion through the humeral head during screw insertion; and 3) achieve an anatomical reduction and rigid fixation. As well as these, they emphasized the need for patients to comply with rehabilitation protocol under the guidance of a trained physiotherapist.

The number of studies reporting the outcomes of T-plate fixations is fewer than that reporting the outcomes of LCP fixations.
The lower reporting is thought to be because the higher rate of fixation loss associated with the former type of plating, which means that surgeons are less likely to choose this method for patients with osteoporosis or severe comminution. Furthermore, because T-plating has been associated with subacromial impingement and screw loosening, other types of plates associated with fewer complications may be preferentially chosen over Tplates.

However, the authors of this study considered that despite the inability of T-LCPs to restore the medial support, unlike LCPs, impacted reduction is only a moderate compromise in comparison to the rigid fixation achieved using T-plates, which provides a large surface area of contact. Therefore, we agreed that T-LCP fixation may be an particularly appropriate treatment modality for elderly patients with proximal humeral fractures.

In this study, we found that the radiological results, in terms of the neck-shaft angle, after T-plate fixation were "fair" or above in all the patients. Although postoperative complications such as reduction loss, nonunion, and avascular necrosis of the humeral head did not occur, there were instances of mild subacromial impingement of the plate, screw loosening, and malunion.

The prevalence of complications observed in this study is either comparable to or lower than those reported in other studies that used either LCP or T-plate fixation. One of the limitations of this study is that we did not perform a comparative analysis against a non-T-plating technique. Moreover, unlike most studies similar to ours, we did not measure functional parameters such as the Constant score. Another limitation is that there were no patients with 4-part fractures; the inadvertent omission of this class of fracture group resulted from the retrospective nature of the study design. Thus, future studies should make a comparative analysis of outcomes after internal fixation using a variety of plating techniques in a larger sample of elderly patients with proximal humeral fractures and make statistical and case analyses of the clinical and functional outcomes to investigate ways to reduce postoperative complications after T-LCP fixation.

\section{Conclusion}

In this study, we observed satisfactory clinical and radiological outcomes after internal fixation using T-LCPs in elderly patients with proximal humeral fractures. Although the locking T-plate fixation may be associated with short malunions, it is useful in that it ensures a large surface area of contact between the plate and fracture and, thereby, a rigid fixation.

\section{References}

1. Court-Brown CM, Caesar B. Epidemiology of adult fractures: a review. Injury. 2006;37(8):691-7.

2. Palvanen M, Kannus P, Niemi S, Parkkari J. Update in the epi- 
demiology of proximal humeral fractures. Clin Orthop Relat Res. 2006;442:87-92.

3. Neer CS 2nd. Displaced proximal humeral fractures. I. Classification and evaluation. J Bone Joint Surg Am. 1970;52(6):107789.

4. Oh $\mathrm{JH}, \mathrm{Kim} \mathrm{YH}$. The current concepts in the treatment of proximal humerus fracture. J Korean Fract Soc. 2012;25(1):94104.

5. Seide K, Triebe J, Faschingbauer M, et al. Locked vs. unlocked plate osteosynthesis of the proximal humerus: a biomechanical study. Clin Biomech (Bristol, Avon). 2007;22(2):176-82.

6. Min WK, Sin SJ, Jeon IH, et al. Fixation failure of LCP during the treatment of proximal humerus fractures. J Korean Fract Soc. 2006;19(2):188-92.

7. Bono CM, Grossman MG, Hochwald N, Tornetta P 3rd. Radial and axillary nerves. Anatomic considerations for humeral fixation. Clin Orthop Relat Res. 2000;(373):259-64.

8. Jiang R, Luo CF, Zeng BF, Mei GH. Minimally invasive plating for complex humeral shaft fractures. Arch Orthop Trauma Surg. 2007;127(7):531-5.

9. Oh $\mathrm{CH}$, Oh JH, Kim SH, Jo KH, Bin SW, Gong HS. Hemiarthoplasty with bone block graft and low profile prosthesis for the comminuted proximal humerus fractures. J Korean Fract Soc. 2008;21(3):213-9.
10. Jung SW. A separate approach and cephalo-diaphyseal plate fixation for the comminuted metadiaphyseal fractures of the proximal humerus. J Korean Fract Soc. 2013;26(1):8-13.

11. Kim DW, Kim CK, Jung SW, Kim HS. Operative treatment of displaced proximal humerus fractures with the angular stable locking compression plate. Clin Should Elbow. 2011;14(1):2734.

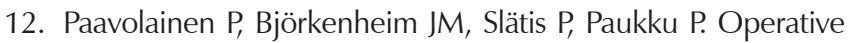
treatment of severe proximal humeral fractures. Acta Orthop Scand. 1983;54(3):374-9.

13. Owsley KC, Gorczyca JT. Fracture displacement and screw cutout after open reduction and locked plate fixation of proximal humeral fractures [corrected]. J Bone Joint Surg Am. 2008;90(2):233-40.

14. Bansal V, Sohal HS, Bhoparai RS. Philos plate in proximal humerus fracture-its functional outcome and complications. Int J Orthop. 2015;2(3):317-22.

15. Sommer C, Babst R, Müller M, Hanson B. Locking compression plate loosening and plate breakage: a report of four cases. J Orthop Trauma. 2004;18(8):571-7.

16. Hussain S, Gul M, Dhar S. Open reduction and internal fixation of displaced proximal humerus fractures with AO stainless steel T-plate. Malays Orthop J. 2014;8(1):8-13. 